DPNU-96-08

hep-ph/9602338

February 1996

\title{
Isospin Breaking Effects in the Anomalous Processes with Vector Mesons
}

\author{
Michio Hashimoto \\ Department of Physics Nagoya University, Nagoya 464-01 Japan
}

\begin{abstract}
We introduce isospin breaking terms as well as $S U(3)$ breaking terms to the anomalous $V V P$ coupling in the hidden local symmetry scheme without affecting the low energy theorem on the processes such as $\pi \rightarrow 2 \gamma$ and $\gamma \rightarrow 3 \pi$. It is shown that the predictions from these terms coincide successfully with all the experimental data of anomalous decays. It is also predicted that the decay widths of $\rho^{0} \rightarrow \pi^{0} \gamma$ and $\phi \rightarrow \eta^{\prime} \gamma$ are $114 \pm 7 \mathrm{keV}$ and $0.552 \pm 0.055 \mathrm{keV}$, respectively.
\end{abstract}

PACS: 12.39.Fe, 12.40.Vv, 13.25.-k, 13.65.+i, 14.40.Aq, 14.65.Bt

\section{Introduction}

Anomalous processes involving vector mesons are interesting probes to test the effective theories of QCD through the low-energy and high-luminosity $e^{+} e^{-}$collider experiments in near future. In particular, the DA $\Phi \mathrm{NE} \phi$-factory is expected to yield $2 \times 10^{10} \phi$-meson decays per year [1], which will provide us with high quality data for decays of pseudoscalar and vector mesons in the light quark sector. We have improved upper bounds of the branching ratios of rare $\phi$-decays such as $\phi \rightarrow \rho \gamma, \phi \rightarrow \omega \gamma$, etc. and may be able to obtain the branching ratio of $\phi \rightarrow \eta^{\prime} \gamma$ [1] for which only the upper bound is known today [2]. Moreover, uncertainty of the data on $\rho^{0} \rightarrow \pi^{0} \gamma$ will be much reduced [1].

These radiative decays are associated with the flavor anomaly of QCD and are described by the Wess-Zumino-Witten(WZW) term[3] in the low energy limit.

\footnotetext{
${ }^{1}$ e-mail address: michioh@eken.phys.nagoya-u.ac.jp
} 
Based on the hidden local symmetry(HLS) [4][5] for the vector mesons, Fujiwara et al.[6] proposed a systematic way to incorporate vector mesons into such a chiral Lagrangian with WZW term without affecting the low-energy theorem on $\pi^{0} \rightarrow 2 \gamma, \gamma \rightarrow 3 \pi$ etc. Bramon et al. [8] studied extensively the radiative vector meson decays by introducing $S U(3)$ breaking into the anomalous Lagrangian of Fujiwara et al.[6]. However, method of Bramon et al. is not consistent with the low-energy theorem, especially on $\eta\left(\eta^{\prime}\right) \rightarrow 2 \gamma$, which are essentially determined by the WZW term. Thus, if isospin breaking effects were introduced through their method, successful low-energy theorem on $\Gamma\left(\pi^{0} \rightarrow 2 \gamma\right)$ and $\Gamma(\gamma \rightarrow 3 \pi)$ would be violated. Furthermore, the breaking effects are important to account for the difference between $\Gamma\left(\rho^{0} \rightarrow \pi^{0} \gamma\right)$ and $\Gamma\left(\rho^{ \pm} \rightarrow \pi^{ \pm} \gamma\right)$.

In this paper, we construct all possible isospin/SU(3)-broken anomalous HLS Lagrangians with the smallest number of derivatives in a manner consistent with the low energy theorem in contrast to Bramon et al.[8]. This is systematically done through spurion method for the breaking term. It is further assumed that we can neglect direct $V P \gamma$ and $V P^{3}$ couplings $(V=$ vector meson, $P=$ pseudoscalar meson) which are absent in the original Lagrangian[6]. Then we find a parameter region which is consistent with all the existing data on radiative decays of vector mesons. Such a parameter region yields some predictions on the decays like $\Gamma\left(\rho^{0} \rightarrow \pi^{0} \gamma\right), \Gamma\left(\phi \rightarrow \eta^{\prime} \gamma\right), \Gamma(\rho \rightarrow \pi \pi \pi)$ and $\Gamma\left(K^{*} \rightarrow K \pi \pi\right)$.

The paper is organized as follows: In section 2, a review of HLS Lagrangian is given for both non-anomalous and anomalous terms. $S U(3)$ breaking terms are introduced into the non-anomalous HLS Lagrangian à la Bando et al.[5]. In section 3, we construct the most general isospin/SU(3)-broken anomalous Lagrangians with the lowest derivatives in a way consistent with the low energy theorem. In section 4, the phenomenological analysis of these Lagrangians will be successfully done for the radiative decays of vector mesons. In section 5 , we make the analysis for the hadronic anomalous decays. Section 6 is devoted to summary.

\section{Hidden Local Symmetry}

Here we give a brief review of HLS approach[7]. A key observation is that the non-linear sigma model based on the manifold $U(3)_{L} \times U(3)_{R} / U(3)_{V}$ is gauge equivalent to another model having a symmetry $\left[U(3)_{L} \times U(3)_{R}\right]_{\text {global }} \times\left[U(3)_{V}\right]_{\text {local }}$. Vector mesons are introduced as the gauge fields of a hidden local symmetry $\left[U(3)_{V}\right]_{\text {local }}$. The photon field is introduced through gauging $\left[U(3)_{L} \times U(3)_{R}\right]_{\text {global }}$.

The HLS Lagrangian is given by [4], [5]:

$$
\begin{aligned}
\mathcal{L} & =\mathcal{L}_{A}+a \mathcal{L}_{V}+\mathcal{L}_{\text {gauge }} \\
\mathcal{L}_{A} & =-\frac{f_{\pi}^{2}}{8} \operatorname{tr}\left(D_{\mu} \xi_{L} \cdot \xi_{L}^{\dagger}-D_{\mu} \xi_{R} \cdot \xi_{R}^{\dagger}\right)^{2}
\end{aligned}
$$




$$
\mathcal{L}_{V}=-\frac{f_{\pi}^{2}}{8} \operatorname{tr}\left(D_{\mu} \xi_{L} \cdot \xi_{L}^{\dagger}+D_{\mu} \xi_{R} \cdot \xi_{R}^{\dagger}\right)^{2}
$$

where $f_{\pi} \simeq 131 \mathrm{MeV}$ is the decay constant of pseudoscalar mesons, $D_{\mu} \xi_{L, R} \equiv$ $\left(\partial_{\mu}-i g V_{\mu}\right) \xi_{L, R}+i e \xi_{L, R} Q \cdot B_{\mu}$, with $Q=\operatorname{diag}\left(\frac{2}{3},-\frac{1}{3},-\frac{1}{3}\right)$, and $V_{\mu}$ and $B_{\mu}$ being the vector mesons and the photon fields, respectively, and $\mathcal{L}_{\text {gauge }}$ is the kinetic terms of $V_{\mu}$ and $B_{\mu}$. Here $g, e$, and $a$ are respectively the hidden gauge coupling, the electron charge and a free parameter not determined by the symmetry considerations alone.

The fields $\xi_{L, R}$ and $V_{\mu}$ transform as follows;

$$
\begin{aligned}
\xi_{L, R}(x) & \rightarrow \xi_{L, R}^{\prime}(x)=h(x) \xi_{L, R}(x) g_{L, R}^{\dagger}(x), \\
V_{\mu}(x) & \rightarrow V_{\mu}^{\prime}(x)=h(x) V_{\mu}(x) h^{\dagger}(x)+i h(x) \partial_{\mu} h^{\dagger}(x)
\end{aligned}
$$

where $h(x) \in\left[U(3)_{V}\right]_{\text {local }}, g_{L, R}(x) \in\left[U(3)_{L, R}\right]_{\text {global }}$. To do a phenomenological analysis, we take unitary gauge:

$$
\begin{aligned}
\xi_{R} & =\xi_{L}^{\dagger}=e^{\frac{i P}{f \pi}}, \\
P & =\left(\begin{array}{ccc}
\frac{\pi^{0}}{\sqrt{2}}+\frac{\eta}{\sqrt{3}}+\frac{\eta^{\prime}}{\sqrt{6}} & \pi^{+} & K^{+} \\
\pi^{-} & -\frac{\pi^{0}}{\sqrt{2}}+\frac{\eta}{\sqrt{3}}+\frac{\eta^{\prime}}{\sqrt{6}} & K^{0} \\
K^{-} & \bar{K}^{0} & -\frac{\eta}{\sqrt{3}}+\sqrt{\frac{2}{3}} \eta^{\prime}
\end{array}\right), \\
V & =\left(\begin{array}{ccc}
\frac{\rho^{0}}{\sqrt{2}}+\frac{\omega}{\sqrt{2}} & \rho^{+} & K^{*+} \\
\rho^{-} & -\frac{\rho^{0}}{\sqrt{2}}+\frac{\omega}{\sqrt{2}} & K^{* 0} \\
K^{*-} & \bar{K}^{* 0} & \phi
\end{array}\right),
\end{aligned}
$$

where we assumed that $\eta_{1}-\eta_{8}$ mixing angle $\left(\theta_{\eta_{1}-\eta_{8}}\right)$ is -19.5 degrees, and $\omega_{1}-\omega_{8}$ mixing angle is the ideal mixing (35 degrees). If we take $a=2$ in (2.1), we have the celebrated KSRF relation $M_{\rho}^{2}=2 f_{\pi}^{2} g^{2}$, universality of the $\rho$-meson coupling and the vector meson dominance for the electromagnetic form factor[4][5].

For obtaining the pseudoscalar meson mass terms, we introduce the quark $\operatorname{mass} \operatorname{matrix}(\mathcal{M})$ as,

$$
\mathcal{L}_{M}=\frac{f_{\pi}^{2} \mu}{2} \operatorname{tr}\left(\xi_{R} \mathcal{M} \xi_{L}^{\dagger}+\xi_{L} \mathcal{M} \xi_{R}^{\dagger}\right)+m_{\eta_{1}}^{2}
$$

where $\mu \mathcal{M}$ is relate to mass of $\pi$, etc. and $m_{\eta_{1}}$ is the mass term of $\eta^{\prime}$ due to $U(1)_{A}$ breaking by gluon anomaly. Analogously, we may add appropriate isospin/SU(3) breaking terms to (2.1) [5],

$$
\begin{aligned}
\Delta \mathcal{L}_{A,(V)} & =-\frac{f_{\pi}^{2}}{8} \operatorname{tr}\left(D_{\mu} \xi_{L} \cdot \epsilon_{A,(V)} \xi_{R}^{\dagger} \pm D_{\mu} \xi_{R} \cdot \epsilon_{A,(V)} \xi_{L}^{\dagger}\right)^{2} \\
\epsilon_{A,(V)} & =\operatorname{diag}\left(0,0, \epsilon_{A,(V)}\right) .
\end{aligned}
$$


Further improvements for (2.1) have been elaborated [10]. Here we will not discuss the non-anomalous sector (2.1) any furthermore, because we are only interested in the anomalous sector. We simply assume that the parameters of the non-anomalous Lagrangian have been arranged so as to reproduce the relevant experimental data. Thus we use the experimental values as inputs from the nonanomalous part.

In addition to (2.1) there exists an anomalous part of the HLS Lagrangian. Fujiwara et al.[6] proposed how to incorporate vector mesons in this part of the Lagrangian without changing the anomaly determined by WZW term[6]. They have given the anomalous action as follows;

$$
\Gamma=\Gamma_{W Z W}+\sum_{i=1}^{4} \int_{M^{4}} c_{i} \mathcal{L}_{i}
$$

where

$$
\begin{aligned}
\Gamma_{W Z W} & =-\frac{i N_{c}}{240 \pi^{2}} \int_{M^{5}} \operatorname{tr}\left[(d U) \cdot U^{\dagger}\right]_{\text {covariantization }}^{5}, \\
\mathcal{L}_{1} & =\operatorname{tr}\left(\hat{\alpha}_{L}^{3} \hat{\alpha}_{R}-\hat{\alpha}_{R}^{3} \hat{\alpha}_{L}\right), \\
\mathcal{L}_{2} & =\operatorname{tr}\left(\hat{\alpha}_{L} \hat{\alpha}_{R} \hat{\alpha}_{L} \hat{\alpha}_{R}\right), \\
\mathcal{L}_{3} & =i \operatorname{tr} F_{V}\left(\hat{\alpha}_{L} \hat{\alpha}_{R}-\hat{\alpha}_{R} \hat{\alpha}_{L}\right), \\
\mathcal{L}_{4} & =\frac{i}{2} \operatorname{tr}\left(\hat{F}_{L}+\hat{F}_{R}\right) \cdot\left(\hat{\alpha}_{L} \hat{\alpha}_{R}-\hat{\alpha}_{R} \hat{\alpha}_{L}\right), \\
\hat{\alpha}_{L, R} & =D \xi_{L, R} \cdot \xi_{L, R}^{\dagger}=d \xi_{L, R} \cdot \xi_{L, R}^{\dagger}-i g V+i e \xi_{L, R} A \xi_{L, R}^{\dagger}, \\
U & =\xi_{L}^{\dagger} \xi_{R}, F_{V}=d V-i g V^{2}, \\
\hat{F}_{L, R} & =\xi_{L, R}\left(d A-i e A^{2}\right) \xi_{L, R}^{\dagger} .
\end{aligned}
$$

Notice that $\mathcal{L}_{1} \sim \mathcal{L}_{4}$ have no contribution to anomalous processes such as $\pi^{0} \rightarrow 2 \gamma$ and $\gamma \rightarrow 3 \pi$ at soft momentum limit, because these Lagrangian are constructed with hidden-gauge covariant blocks such as $\hat{\alpha}_{L, R}, F_{V}, \hat{F}_{L, R}[6]$.

We take $c_{3}=c_{4}=-15 C, c_{1}-c_{2}=15 C$ in (2.12) for phenomenological reason[6]. Then we obtained the Lagrangian of anomalous sector as follows:

$$
\begin{aligned}
\mathcal{L}_{\mathrm{FKTUY}} & =5 C\left[3(V V P)-2\left(\gamma P^{3}\right)\right]+\cdots, \\
(V V P) & =-\frac{2 i g^{2}}{f_{\pi}} \operatorname{tr}(V d V d P+d V V d P), \\
\left(\gamma P^{3}\right) & =\frac{4 e}{f_{\pi}^{3}} \operatorname{tr} A(d P)^{3}, \\
C & =-\frac{i N_{c}}{240 \pi^{2}}
\end{aligned}
$$


Here, it is important that the amplitude such as $\pi^{0} \rightarrow 2 \gamma, \gamma \rightarrow 3 \pi$ at low energy limit are determined essentially only by the non-Abelian anomaly of the chiral $U(3)_{L} \times U(3)_{R}$ symmetry. Eq. (2.21) is, of course, consistent with the low energy theorem related to the anomaly.

\section{Isospin/ $S U(3)$-breaking Terms in the Anoma- lous Sector}

We now consider how to modify $\mathcal{L}_{1} \sim \mathcal{L}_{4}$ by introducing isospin/SU(3)-breaking parameters, $\epsilon$ 's, treated as "spurions" [11]. The spurion $\epsilon$ transforms as $\epsilon \rightarrow$ $g_{L}(x) \epsilon g_{R}^{\dagger}(x)$. Then we define the hidden-gauge covariant block $\hat{\epsilon} \equiv \frac{1}{2}\left(\xi_{L} \epsilon \xi_{R}^{\dagger}+\right.$ $\left.\xi_{R} \epsilon^{\dagger} \xi_{L}^{\dagger}\right)$. We construct Lagrangians out of the hidden-gauge covariant blocks such as $\hat{\alpha}_{L, R}, F_{V}, \hat{F}_{L, R}$ and $\hat{\epsilon}$ so as to make them " invariant" under $\left[U(3)_{L} \times\right.$ $\left.U(3)_{R}\right]_{\text {global }} \times\left[U(3)_{V}\right]_{\text {local }}$ as well as parity-, charge conjugation-, and $C P$-transformations. After hidden-gauge fixing, they become explicit breaking terms of the $S U(3)$ symmetry. Then, in general, we obtain isospin/SU(3)-broken anomalous Lagrangians.

$$
\begin{aligned}
\Delta \mathcal{L}_{1}= & \operatorname{tr}\left[\hat{\alpha}_{L}^{3}\left(\hat{\alpha}_{R} \cdot \hat{\epsilon}^{(1)}+\hat{\epsilon}^{(1)} \cdot \hat{\alpha}_{R}\right)-\hat{\alpha}_{R}^{3}\left(\hat{\alpha}_{L} \cdot \hat{\epsilon}^{(1)}+\hat{\epsilon}^{(1)} \cdot \hat{\alpha}_{L}\right)\right] \\
\Delta \mathcal{L}_{1}^{\prime}= & \operatorname{tr}\left(\hat{\alpha}_{L} \hat{\epsilon}^{\left(1^{\prime}\right)} \hat{\alpha}_{L}^{2} \hat{\alpha}_{R}-\hat{\alpha}_{R} \hat{\epsilon}^{\left(1^{\prime}\right)} \hat{\alpha}_{R}^{2} \hat{\alpha}_{L}+\hat{\alpha}_{L}^{2} \hat{\epsilon}^{\left(1^{\prime}\right)} \hat{\alpha}_{L} \hat{\alpha}_{R}-\hat{\alpha}_{R}^{2} \hat{\epsilon}^{\left(1^{\prime}\right)} \hat{\alpha}_{R} \hat{\alpha}_{L}\right), \\
\Delta \mathcal{L}_{2}= & \operatorname{tr}\left(\hat{\epsilon}^{(2)} \cdot \hat{\alpha}_{L}+\hat{\alpha}_{L} \cdot \hat{\epsilon}^{(2)}\right) \hat{\alpha}_{R} \hat{\alpha}_{L} \hat{\alpha}_{R} \\
\Delta \mathcal{L}_{3}= & i \operatorname{tr}\left(F_{V} \cdot \hat{\epsilon}^{(3)}+\hat{\epsilon}^{(3)} \cdot F_{V}\right) \cdot\left(\hat{\alpha}_{L} \hat{\alpha}_{R}-\hat{\alpha}_{R} \hat{\alpha}_{L}\right) \\
\Delta \mathcal{L}_{3}^{\prime}= & i \operatorname{tr} F_{V}\left(\hat{\alpha}_{L} \hat{\epsilon}^{\left(3^{\prime}\right)} \hat{\alpha}_{R}-\hat{\alpha}_{R} \hat{\epsilon}^{\left(3^{\prime}\right)} \hat{\alpha}_{L}\right) \\
\Delta \mathcal{L}_{4}= & i \operatorname{tr}\left[\left(\hat{F}_{L} \cdot \hat{\epsilon}^{(4)}+\hat{\epsilon}^{(4)} \cdot \hat{F}_{L}\right) \cdot\left(\hat{\alpha}_{L} \hat{\alpha}_{R}-\hat{\alpha}_{R} \hat{\alpha}_{L}\right)\right. \\
& \left.+\left(\hat{F}_{R} \cdot \hat{\epsilon}^{(4)}+\hat{\epsilon}^{(4)} \cdot \hat{F}_{R}\right) \cdot\left(\hat{\alpha}_{L} \hat{\alpha}_{R}-\hat{\alpha}_{R} \hat{\alpha}_{L}\right)\right] \\
\Delta \mathcal{L}_{4}^{\prime}= & i \operatorname{tr}\left(\hat{F}_{L}+\hat{F}_{R}\right) \cdot\left(\hat{\alpha}_{L} \hat{\epsilon}^{\left(4^{\prime}\right)} \hat{\alpha}_{R}-\hat{\alpha}_{R} \hat{\epsilon}^{\left(4^{\prime}\right)} \hat{\alpha}_{L}\right) \\
\Delta \mathcal{L}_{5}= & \operatorname{tr}\left(\hat{\alpha}_{L}^{2} \hat{\epsilon}^{(5)} \hat{\alpha}_{R}^{2}-\hat{\alpha}_{R}^{2} \hat{\epsilon}^{(5)} \hat{\alpha}_{L}^{2}\right) \\
\Delta \mathcal{L}_{6}= & i \operatorname{tr}\left(\hat{\epsilon}^{(6)} F_{V}-F_{V} \hat{\epsilon}^{(6)}\right) \cdot\left(\hat{\alpha}_{L}^{2}-\hat{\alpha}_{R}^{2}\right) \\
\Delta \mathcal{L}_{7}= & i \operatorname{tr}\left[\left(\hat{\epsilon}^{(7)} \hat{F}_{L}-\hat{F}_{L} \hat{\epsilon}^{(7)}\right) \hat{\alpha}_{R}^{2}-\left(\hat{\epsilon}^{(7)} \hat{F}_{R}-\hat{F}_{R} \hat{\epsilon}^{(7)}\right) \hat{\alpha}_{L}^{2}\right] \\
\Delta \mathcal{L}_{8}= & i \operatorname{tr}\left[\left(\hat{\epsilon}^{(8)} \hat{F}_{L}-\hat{F}_{L} \hat{\epsilon}^{(8)}\right) \hat{\alpha}_{L}^{2}-\left(\hat{\epsilon}^{(8)} \hat{F}_{R}-\hat{F}_{R} \hat{\epsilon}^{(8)}\right) \hat{\alpha}_{R}^{2}\right]
\end{aligned}
$$

Here $\hat{\alpha}_{L, R}, F_{V}, \hat{F}_{L, R}$ transform under $P$ and $C$ transformations as

$$
\begin{array}{ll}
P: & \hat{\alpha}_{L, R \mu} \longrightarrow \hat{\alpha}_{R, L}^{\mu}, \\
& F_{V \mu \nu} \longrightarrow F_{V}^{\mu \nu}, \hat{F}_{L, R \mu \nu} \longrightarrow \hat{F}_{R, L}^{\mu \nu}, \\
C: \quad & \hat{\alpha}_{L, R} \longrightarrow-\hat{\alpha}_{R, L}^{T}, \\
& F_{V} \longrightarrow-F_{V}^{T}, \hat{F}_{L, R} \longrightarrow-\hat{F}_{R, L}^{T} .
\end{array}
$$

We could introduce $P$-odd "spurion" $\hat{\epsilon}_{-}=\frac{1}{2}\left(\xi_{L} \epsilon \xi_{R}^{\dagger}-\xi_{R} \epsilon^{\dagger} \xi_{L}^{\dagger}\right)$, which, however, is not relevant to the following analysis. 
Among the above additional terms, only $\Delta \mathcal{L}_{3,4,6,7,8}$ terms contribute to the radiative decays of vector mesons. There still exists too many parameters. However, we may select the combination of $\Delta \mathcal{L}_{1 \sim 8}$ so as to eliminate the direct $V \gamma P$-, $V P^{3}$-coupling terms, which do not exist in the original Lagrangian $\mathcal{L}_{\text {FKTUY }}$ (2. 21). Then the isospin/SU(3)-broken anomalous Lagrangians consist of only the following two terms:

$$
\begin{aligned}
-\Delta \mathcal{L}_{V V P}^{a}= & \frac{3 g^{2}}{4 \pi^{2} f_{P}} \operatorname{tr} \epsilon^{\prime}(d V d V P+P d V d V)-\frac{3 e^{2}}{4 \pi^{2} f_{P}} \operatorname{tr} \epsilon^{\prime}(d A d A P+P d A d A) \\
& +i \frac{3 e}{4 \pi^{2} f_{P}^{3}} \operatorname{tr} \epsilon^{\prime}\left(d P^{3} A-A d P^{3}+d P A d P^{2}-d P^{2} A d P\right), \\
-\Delta \mathcal{L}_{V V P}^{b}= & \frac{3 g^{2}}{2 \pi^{2} f_{P}} \operatorname{tr} \epsilon(d V P d V)-\frac{3 e^{2}}{2 \pi^{2} f_{P}} \operatorname{tr} \epsilon(d A P d A) \\
& +i \frac{3 e}{2 \pi^{2} f_{P}^{3}} \operatorname{tr} \epsilon\left(d P^{3} A-A d P^{3}\right) .
\end{aligned}
$$

Our $\Delta \mathcal{L}_{V V P}^{b}$ resembles the $S U(3)$-broken anomalous Lagrangian introduced by Bramon et al.[8], but, is conceptually quite different from the latter. In fact the prediction on $\eta\left(\eta^{\prime}\right) \rightarrow 2 \gamma$ decay width in the latter is different from the low energy theorem's prediction. On the other hand, our $\Delta \mathcal{L}_{V V P}^{a, b}$ do not change the low energy theorem by construction obviously.

\section{Phenomenological Analysis for Radiative De- cays}

We now discuss the phenomenological consequences of our Lagrangian $\mathcal{L}_{\text {anomalous }}=$ $\mathcal{L}_{\text {FKTUY }}+\Delta \mathcal{L}_{V V P}^{a}+\Delta \mathcal{L}_{V V P}^{b}$. For convenience, we define relevant coupling constant as

$$
g_{V P \gamma}=\sum_{V^{\prime}} \frac{g_{V V^{\prime} P} g_{V^{\prime} \gamma}}{M_{V^{\prime}}^{2}}
$$

considering that these decays proceed via intermediate vector mesons $V^{\prime}$. Then we obtain each radiative decay width

$$
\begin{aligned}
\Gamma(V \longrightarrow P \gamma) & =\frac{1}{3} \alpha \cdot g_{V P \gamma}^{2}\left(\frac{M_{V}^{2}-M_{P}^{2}}{2 M_{V}}\right)^{3}, \\
\Gamma\left(\eta^{\prime} \longrightarrow V \gamma\right) & =\alpha \cdot g_{\eta^{\prime} V \gamma}^{2}\left(\frac{M_{\eta^{\prime}}^{2}-M_{V}^{2}}{2 M_{\eta^{\prime}}}\right)^{3},
\end{aligned}
$$

where $g_{V^{\prime} P}, g_{V^{\prime} \gamma}$, and $M_{V^{\prime}}$ are anomalous $V V^{\prime} P$ coupling constant, $V^{\prime}-\gamma$ mixing, and mass of vector meson, respectively. In $\Delta \mathcal{L}_{V V P}^{a, b}$ we take a parametrization for 
convenience:

$$
\epsilon^{\prime}=\left(\begin{array}{ccc}
-\epsilon_{1}^{\prime} & & \\
& -\epsilon_{2}^{\prime} & \\
& & -\epsilon_{3}^{\prime}
\end{array}\right), \epsilon=\left(\begin{array}{ccc}
\epsilon_{1}+\epsilon_{1}^{\prime} & & \\
& \epsilon_{2}+\epsilon_{2}^{\prime} & \\
& & \epsilon_{3}+\epsilon_{3}^{\prime}
\end{array}\right) .
$$

Thus each $g_{V P \gamma}$ is given in terms of the parameters in $\Delta \mathcal{L}_{V V P}^{a, b}$

$$
\left\{\begin{aligned}
g_{\rho^{0} \pi^{0} \gamma} & =G\left(1+4 \epsilon_{1}-2 \epsilon_{2}+3 \delta\right), \\
g_{\rho^{ \pm} \pi^{ \pm} \gamma} & =G\left(1+3 \epsilon_{1}^{\prime}-3 \epsilon_{2}^{\prime}+4 \epsilon_{1}-2 \epsilon_{2}\right), \\
g_{\omega \pi^{0} \gamma} & =3 G\left(1+\frac{4}{3} \epsilon_{1}+\frac{2}{3} \epsilon_{2}-\frac{\delta}{3}\right), \\
g_{\omega \eta \gamma} & =\frac{f_{\pi}}{f_{\eta}} \sqrt{\frac{2}{3}} G\left(1+4 \epsilon_{1}-2 \epsilon_{2}-\sqrt{2} \theta_{V}-3 \delta-\frac{\theta_{P}}{\sqrt{2}}\right), \\
g_{\rho^{0} \eta \gamma} & =\frac{f_{\pi}}{f_{\eta}} \sqrt{6} G\left(1+\frac{4}{3} \epsilon_{1}+\frac{2}{3} \epsilon_{2}+\frac{\delta}{3}-\frac{\theta_{P}}{\sqrt{2}}\right), \\
g_{\phi \eta \gamma} & =\frac{f_{\pi}}{f_{\eta}} \frac{2}{\sqrt{3}} G\left(1+2 \epsilon_{3}+\frac{\theta_{V}}{\sqrt{2}}+\sqrt{2} \theta_{P}\right), \\
g_{K^{* \pm} K^{ \pm} \gamma} & =\frac{f_{\pi}}{f_{K}} G\left(1+3 \epsilon_{1}^{\prime}-3 \epsilon_{3}^{\prime}+4 \epsilon_{1}-2 \epsilon_{3}\right), \\
g_{K^{*} \bar{K}^{0} \gamma} & =-\frac{f_{\pi}}{f_{K}} 2 G\left(1+\epsilon_{2}+\epsilon_{3}\right), \\
g_{\phi \pi^{0} \gamma} & =g_{\omega \pi^{0} \gamma} \cdot \theta_{V}, \\
g_{\eta^{\prime} \rho^{0} \gamma} & =\frac{f_{\pi}}{f_{\eta^{\prime}}} \sqrt{3} G\left(1+\frac{4}{3} \epsilon_{1}+\frac{2}{3} \epsilon_{2}+\frac{\delta}{3}+\sqrt{2} \theta_{P}\right), \\
g_{\eta^{\prime} \omega \gamma} & =\frac{f_{\pi}}{f_{\eta^{\prime}}} \frac{1}{\sqrt{3}} G\left(1+4 \epsilon_{1}-2 \epsilon_{2}+2 \sqrt{2} \theta_{V}-3 \delta+\sqrt{2} \theta_{P}\right), \\
g_{\phi \eta^{\prime} \gamma} & =-\frac{f_{\pi}}{f_{\eta^{\prime}}} \frac{2 \sqrt{2}}{\sqrt{3}} G\left(1+2 \epsilon_{3}-\frac{\theta_{V}}{2 \sqrt{2}}-\frac{\theta_{P}}{\sqrt{2}}\right),
\end{aligned}\right.
$$

where $G=\frac{g}{4 \pi^{2} f_{\pi}}$.

The parameters $\theta_{V}, \theta_{P}$ appearing in the expression of $g_{V P \gamma}$ stand for the difference of $\phi-\omega, \eta-\eta^{\prime}$ mixing angles from the already determined mixing angles by the non-anomalous sector, i.e. , ideal mixing and $\eta_{1}-\eta_{8}$ mixing, respectively. The parameter $\delta$ comes from the $\rho$ - $\omega$ interference effect arising from the small mass difference of $\rho$ and $\omega$.

2For reproducing the experimental value of $\Gamma(\phi \rightarrow \rho \pi \rightarrow \pi \pi \pi)$, we took $\theta_{V}=0.0600 \pm 0.0017$. The sign comes from the observed $\phi$ - $\omega$ interference effects in $e^{+} e^{-} \rightarrow \pi^{+} \pi^{-} \pi^{0}[2]$.

Similarly, we consider the decay of $\omega \rightarrow \pi \pi$, which is $G$-parity violating process. If the isospin were not broken, such process would not exist. The experimental value of $\Gamma(\omega \rightarrow \pi \pi)$ is reproduced for $\delta=0.0348 \pm 0.0024$. We calculated $\delta$ from $\Gamma(\omega \rightarrow \pi \pi) / \Gamma(\rho \rightarrow \pi \pi)=\delta^{2} \cdot \frac{p_{\omega \rightarrow \pi \pi}^{3}}{M_{\omega}^{2}} / \frac{p_{\rho \rightarrow \pi \pi}^{3}}{M_{\rho}^{2}}$, where $p_{V \rightarrow \pi \pi}$ is the final state pion momentum. The ambiguity of the sign has been resolved recently through the decays of $\omega$ produced in $\pi^{-} p \rightarrow \omega n$ [9], in which the constructive interference has been supported.

The mixing angle $\theta_{\eta_{1}-\eta_{8}}(=\arcsin (-1 / 3))$ has been deduced from $\eta$ - $\eta^{\prime}$ phenomenology[12]. Thus we take $\theta_{P}=0$.

There are essentially five free parameters from $\Delta \mathcal{L}_{V V P}^{a, b}$ in 4.5 , because $\epsilon_{1}^{\prime}$ is negligible. We search for the parameter region consistent with the experiments. 


\begin{tabular}{|c|c|c|c|c|}
\hline Decay Mode & (I) $\mathcal{L}_{\text {FKTUY }}[6]$ & (II) Bramon's [8] & (III) Ours & exp.[2] \\
\hline$\overline{\bar{\Gamma}\left(\rho^{0} \rightarrow \pi^{0} \gamma\right)}$ & $86.2 \pm 0.8 \mathrm{keV}$ & $86.2 \pm 0.8 \mathrm{keV}$ & $\overline{114 \pm 7 \mathrm{keV}}$ & $\overline{121 \pm 31 \mathrm{keV}}$ \\
\hline$\Gamma\left(\rho^{ \pm} \rightarrow \pi^{ \pm} \gamma\right)$ & $85.6 \pm 0.8 \mathrm{keV}$ & $85.6 \pm 0.8 \mathrm{keV}$ & $68.1 \pm 0.6 \mathrm{keV}$ & $68 \pm 7 \mathrm{keV}$ \\
\hline$\Gamma\left(\omega \rightarrow \pi^{0} \gamma\right)$ & $815 \pm 8 \mathrm{keV}$ & $815 \pm 8 \mathrm{keV}$ & $745 \pm 31 \mathrm{keV}$ & $717 \pm 43 \mathrm{keV}$ \\
\hline$\Gamma(\omega \rightarrow \eta \gamma)$ & $6.68 \pm 0.59 \mathrm{keV}$ & $5.6 \pm 0.6 \mathrm{keV}$ & $4.94 \pm 0.75 \mathrm{keV}$ & $7.00 \pm 1.77 \mathrm{keV}$ \\
\hline$\Gamma\left(\rho^{0} \rightarrow \eta \gamma\right)$ & $52.4 \pm 4.6 \mathrm{keV}$ & $52.4 \pm 4.6 \mathrm{keV}$ & $50.4 \pm 6.0 \mathrm{keV}$ & $57.5 \pm 10.6 \mathrm{keV}$ \\
\hline$\Gamma(\phi \rightarrow \eta \gamma)$ & $80.7 \pm 7.1 \mathrm{keV}$ & $57 \pm 9 \mathrm{keV}$ & $62.8 \pm 8.8 \mathrm{keV}$ & $56.9 \pm 2.9 \mathrm{keV}$ \\
\hline$\Gamma\left(K_{-}^{* \pm} \rightarrow K^{ \pm} \gamma\right)$ & $32.8 \pm 0.9 \mathrm{keV}$ & $47 \pm 5 \mathrm{keV}$ & $50.4 \pm 1.7 \mathrm{keV}$ & $50 \pm 5 \mathrm{keV}$ \\
\hline$\Gamma\left(\overline{K^{* 0}} \rightarrow \bar{K}^{0} \gamma\right)$ & $132 \pm 4 \mathrm{keV}$ & $107 \pm 15 \mathrm{keV}$ & $106.9 \pm 5.5 \mathrm{keV}$ & $117 \pm 10 \mathrm{keV}$ \\
\hline$\Gamma\left(\phi \rightarrow \pi^{0} \gamma\right)$ & ---- & $6.76 \pm 0.34 \mathrm{keV}$ & $6.19 \pm 0.55 \mathrm{keV}$ & $5.80 \pm 0.58 \mathrm{keV}$ \\
\hline$\Gamma\left(\eta^{\prime} \rightarrow \rho^{0} \gamma\right)$ & $61.7 \pm 2.7 \mathrm{keV}$ & $61.7 \pm 2.7 \mathrm{keV}$ & $59.3 \pm 4.5 \mathrm{keV}$ & $61 \pm 5 \mathrm{keV}$ \\
\hline$\Gamma\left(\eta^{\prime} \rightarrow \omega \gamma\right)$ & $5.74 \pm 0.25 \mathrm{keV}$ & $7.86 \pm 0.34 \mathrm{keV}$ & $7.14 \pm 0.71 \mathrm{keV}$ & $6.1 \pm 0.8 \mathrm{keV}$ \\
\hline$\Gamma\left(\phi \rightarrow \eta^{\prime} \gamma\right)$ & $0.827 \pm 0.036 \mathrm{keV}$ & $0.5 \pm 0.1 \mathrm{keV}$ & $0.552 \pm 0.055 \mathrm{keV}$ & $<1.84 \mathrm{keV}$ \\
\hline$\overline{\Gamma\left(\pi^{0} \rightarrow 2 \gamma\right)}$ & $\begin{array}{ll}7.70 \mathrm{eV}\end{array}$ & $\begin{array}{l}7.70 \mathrm{eV} \\
\end{array}$ & $\begin{array}{ll}7.70 \mathrm{eV} \\
\end{array}$ & 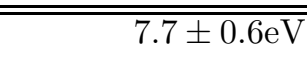 \\
\hline$\Gamma(\eta \rightarrow 2 \gamma)$ & $0.46 \pm 0.04 \mathrm{keV}$ & $0.51 \pm 0.04 \mathrm{keV}$ & $0.46 \pm 0.04 \mathrm{keV}$ & $0.46 \pm 0.04 \mathrm{keV}$ \\
\hline$\Gamma\left(\eta^{\prime} \rightarrow 2 \gamma\right)$ & $4.26 \pm 0.19 \mathrm{keV}$ & $3.6 \pm 0.2 \mathrm{keV}$ & $4.26 \pm 0.19 \mathrm{keV}$ & $4.26 \pm 0.19 \mathrm{keV}$ \\
\hline$\overline{\bar{\Gamma}\left(\eta \rightarrow \pi^{+} \pi^{-} \gamma\right)}$ & 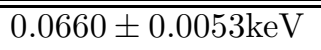 & 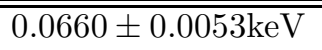 & $\overline{0.0648 \pm 0.0059 \mathrm{keV}}$ & 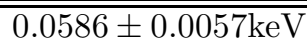 \\
\hline$\Gamma\left(\eta^{\prime} \rightarrow \pi^{+} \pi^{-} \gamma\right)$ & $53.0 \pm 2.2 \mathrm{keV}$ & $53.0 \pm 2.2 \mathrm{keV}$ & $50.3 \pm 3.4 \mathrm{keV}$ & $56.1 \pm 6.4 \mathrm{keV}$ \\
\hline
\end{tabular}

Table 1: Radiative Decay Width of Vector Mesons

(I) Values of original $\mathcal{L}_{\text {FKTUY }}$ [6] (II) Values of the $S U(3)$-broken model by Bramon et al.[8]

$\left(\epsilon_{3}=-0.1 \pm 0.03\right)$. The predictions by this model to $\eta\left(\eta^{\prime}\right) \rightarrow 2 \gamma$ differ from the experimental value.

(III) Values of our model. The region of parameters : $0.0279<4 \epsilon_{1}-2 \epsilon_{2}<0.0670$,

$-0.0471<\frac{4}{3} \epsilon_{1}+\frac{2}{3} \epsilon_{2}<-0.0174,-0.112<\epsilon_{2}+\epsilon_{3}<-0.0902,-0.0925<\epsilon_{3}<-0.0702$,

$4 \epsilon_{1}-2 \epsilon_{2}-3 \epsilon_{2}^{\prime}=-0.108,4 \epsilon_{1}-2 \epsilon_{3}-3 \epsilon_{3}^{\prime}=0.235$, (We took the center value to $\epsilon^{\prime}$ ).

We take $g=4.27 \pm 0.02$ from $\Gamma(\rho \rightarrow \pi \pi)=151.2 \pm 1.2 \mathrm{MeV}$, and $f_{\pi}=$ $131 \mathrm{MeV}, f_{K}=160 \pm 2 \mathrm{MeV}[2]$, and $f_{\eta}=150 \pm 6 \mathrm{MeV}, f_{\eta^{\prime}}=142 \pm 3 \mathrm{MeV}$ from $\eta\left(\eta^{\prime}\right) \rightarrow 2 \gamma[2]$. Then we obtained the results listed in Table 1 .

In Table 1, (I) (III) mean:

(I) Values of original $\mathcal{L}_{\text {FKTUY }}$,

(II) Values of the $S U(3)$-broken model by Bramon et al. [8] $\left(\epsilon_{3}=-0.1 \pm 0.03\right)$

The predictions by this model to $\eta\left(\eta^{\prime}\right) \rightarrow 2 \gamma$ differ from the experimental value,

(III) Values of our model

The region of parameters $0.0279<4 \epsilon_{1}-2 \epsilon_{2}<0.0670$,

$-0.0471<\frac{4}{3} \epsilon_{1}+\frac{2}{3} \epsilon_{2}<-0.0174,-0.112<\epsilon_{2}+\epsilon_{3}<-0.0902$,

$-0.0925<\epsilon_{3}<-0.0702,4 \epsilon_{1}-2 \epsilon_{2}-3 \epsilon_{2}^{\prime}=-0.108,4 \epsilon_{1}-2 \epsilon_{3}-3 \epsilon_{3}^{\prime}=0.235$,

( We took the center value to $\epsilon^{\prime}$ ).

The parameter region (III) suggests that isospin/SU(3)-breaking effects for the anomalous sector cannot be given by the quark mass matrix in a simple manner. 
The results for $\Gamma\left(\rho^{0} \rightarrow \pi^{0} \gamma\right), \Gamma\left(\rho^{ \pm} \rightarrow \pi^{ \pm} \gamma\right), \Gamma\left(\omega \rightarrow \pi^{0} \gamma\right), \Gamma(\omega \rightarrow 3 \pi)$ in Table 1 suggest that isospin breaking terms are very important. Both (I) and (II) in Table 1 do not have isospin breaking terms. These values differ substantially from the experiments, which cannot be absorbed by the ambiguity of the hidden-gauge coupling $g$ whose value are determined either by $\Gamma(\rho \rightarrow 2 \pi)$ or by $\Gamma\left(\rho \rightarrow e^{+} e^{-}\right)$. In order to avoid this ambiguity, let us take some expressions cancelling $g$, i.e. , $\Gamma(\rho \rightarrow \pi \gamma) / \Gamma(\rho \rightarrow 2 \pi), \Gamma(\omega \rightarrow \pi \gamma) / \Gamma(\rho \rightarrow 2 \pi)$. Then we find that predictions of the original $\mathcal{L}_{\text {FKTUY }}$ and Bramon et al.[8] are still different from the experiments. These Lagrangian without isospin breaking terms yields

$$
\begin{aligned}
\frac{\Gamma\left(\rho^{0} \rightarrow \pi \gamma\right)}{\Gamma(\rho \rightarrow 2 \pi)}= & \alpha M_{\rho}^{2} P_{\rho \rightarrow \pi \gamma}^{3} / 16 \pi^{3} f_{\pi}^{2} P_{\rho \rightarrow \pi \pi}^{3}, \\
= & 5.6 \times 10^{-4}, \\
& {\left[\exp .(7.9 \pm 2.0) \times 10^{-4}\right], } \\
& {\left[\text { ours }(7.5 \pm 0.5) \times 10^{-4}\right], } \\
\frac{\Gamma\left(\rho^{ \pm} \rightarrow \pi \gamma\right)}{\Gamma(\rho \rightarrow 2 \pi)}= & \alpha M_{\rho}^{2} P_{\rho \rightarrow \pi \gamma}^{3} / 16 \pi^{3} f_{\pi}^{2} P_{\rho \rightarrow \pi \pi}^{3}, \\
= & 5.6 \times 10^{-4} \\
& {\left[\exp .(4.5 \pm 0.5) \times 10^{-4}\right], } \\
& {\left[\text { ours } 4.5 \times 10^{-4}\right], } \\
\frac{\Gamma(\omega \rightarrow \pi \gamma)}{\Gamma(\rho \rightarrow 2 \pi)}= & 9 \alpha M_{\rho}^{2} P_{\rho \rightarrow \pi \gamma}^{3} / 16 \pi^{3} f_{\pi}^{2} P_{\rho \rightarrow \pi \pi}^{3}, \\
= & 5.4 \times 10^{-3}, \\
& {\left[\exp .(4.7 \pm 0.4) \times 10^{-3}\right], } \\
& {\left[\text { ours }(4.9 \pm 0.2) \times 10^{-3}\right] . }
\end{aligned}
$$

Finally, we pay attention to $\Gamma\left(\eta\left(\eta^{\prime}\right) \rightarrow \pi^{+} \pi^{-} \gamma\right)$, which are given by

$$
\begin{aligned}
\Gamma\left(\eta \rightarrow \pi^{+} \pi^{-} \gamma\right)= & \frac{3 g^{2} \alpha}{16 \pi^{6} f_{\eta}^{2} M_{\eta}} \int d E_{+} d E_{-}\left[\boldsymbol{p}_{+}^{2} \boldsymbol{p}_{-}^{2}-\left(\boldsymbol{p}_{+} \cdot \boldsymbol{p}_{-}\right)^{2}\right] \times \\
& \left(\frac{1+4 / 3 \epsilon_{1}+2 / 3 \epsilon_{2}}{\left(p_{+}+p_{-}\right)^{2}-M_{\rho}^{2}}+\frac{1+4 \epsilon_{1}+2 \epsilon_{2}}{3 M_{\rho}^{2}}\right)^{2} \\
\Gamma\left(\eta^{\prime} \rightarrow \pi^{+} \pi^{-} \gamma\right)= & \frac{3 g^{2} \alpha}{32 \pi^{6} f_{\eta^{\prime}}^{2} M_{\eta^{\prime}}} \int d E_{+} d E_{-}\left[\boldsymbol{p}_{+}^{2} \boldsymbol{p}_{-}^{2}-\left(\boldsymbol{p}_{+} \cdot \boldsymbol{p}_{-}\right)^{2}\right] \times \\
& \left(\frac{1+4 / 3 \epsilon_{1}+2 / 3 \epsilon_{2}}{\left(p_{+}+p_{-}\right)^{2}+i M_{\rho} \Gamma_{\rho}-M_{\rho}^{2}}+\frac{1+4 \epsilon_{1}+2 \epsilon_{2}}{3 M_{\rho}^{2}}\right)^{2} \\
\Gamma_{\rho}= & \Gamma(\rho \rightarrow 2 \pi) \cdot\left(\frac{\left(q_{\pi^{+}}+q_{\pi^{-}}\right)^{2}-4 M_{\pi}^{2}}{M_{\rho}^{2}-4 M_{\pi}^{2}}\right)^{3 / 2} \theta\left(\left(q_{\pi^{+}}+q_{\pi^{-}}\right)^{2}-4 M_{\pi}^{2}\right),
\end{aligned}
$$




\begin{tabular}{lrrrr}
\hline Decay Mode & $(\mathrm{I}) \mathcal{L}_{\text {FKTUY }}[6]$ & (II) Bramon's [8] & (III) Ours & exp. [2] \\
\hline \hline$\Gamma\left(\omega \rightarrow \pi^{0} \pi^{+} \pi^{-}\right)$ & $8.18 \pm 0.23 \mathrm{MeV}$ & $8.18 \pm 0.23 \mathrm{MeV}$ & $7.62 \pm 0.26 \mathrm{MeV}$ & $7.49 \pm 0.12 \mathrm{MeV}$ \\
\hline$\Gamma\left(\rho^{0} \rightarrow \pi^{0} \pi^{+} \pi^{-}\right)$ & ---- & ---- & $6.70 \pm 3.25 \mathrm{keV}$ & $<18 \mathrm{keV}$ \\
$\Gamma\left(\rho^{ \pm} \rightarrow \pi^{ \pm} \pi^{0} \pi^{0}\right)$ & ---- & ---- & $4.76 \pm 1.70 \mathrm{keV}$ & ---- \\
$\Gamma\left(\rho^{ \pm} \rightarrow \pi^{ \pm} \pi^{+} \pi^{-}\right)$ & ---- & ---- & $0.125 \pm 0.121 \mathrm{keV}$ & ----- \\
$\Gamma\left(K^{*-} \rightarrow \bar{K}^{0} \pi^{0} \pi^{-}\right)$ & $17.9 \pm 1.6 \mathrm{keV}$ & $14 \pm 2 \mathrm{keV}$ & $12.3 \pm 1.1 \mathrm{keV}$ & $<35 \mathrm{keV}$ \\
$\Gamma\left(K^{*-} \rightarrow K^{-} \pi^{+} \pi^{-}\right)$ & $8.65 \pm 0.33 \mathrm{keV}$ & $6.6 \pm 0.9 \mathrm{keV}$ & $6.26 \pm 0.55 \mathrm{keV}$ & $<40 \mathrm{keV}$ \\
$\Gamma\left(K^{*-} \rightarrow K^{-} \pi^{0} \pi^{0}\right)$ & $1.11 \pm 0.04 \mathrm{keV}$ & $0.72 \pm 0.06 \mathrm{keV}$ & $0.685 \pm 0.081 \mathrm{keV}$ & ----- \\
$\Gamma\left(\bar{K}^{* 0} \rightarrow K^{-} \pi^{0} \pi^{+}\right)$ & $23.2 \pm 2.1 \mathrm{keV}$ & $18 \pm 3 \mathrm{keV}$ & $16.8 \pm 1.6 \mathrm{keV}$ & ----- \\
$\Gamma\left(\bar{K}^{* 0} \rightarrow \bar{K}^{0} \pi^{-} \pi^{+}\right)$ & $9.04 \pm 0.83 \mathrm{keV}$ & $7.0 \pm 0.1 \mathrm{keV}$ & $6.26 \pm 0.60 \mathrm{keV}$ & $<35 \mathrm{keV}$ \\
$\Gamma\left(\bar{K}^{* 0} \rightarrow \bar{K}^{0} \pi^{0} \pi^{0}\right)$ & $1.11 \pm 0.05 \mathrm{keV}$ & $0.71 \pm 0.06 \mathrm{keV}$ & $0.522 \pm 0.05 \mathrm{keV}$ & ----- \\
\hline \hline
\end{tabular}

Table 2: Hadronic Decay Width of Vector Mesons

(I) Values of original $\mathcal{L}_{\text {FKTUY }}$ (II) Values of the $S U(3)$-broken model by Bramon et al.[8] $\left(\epsilon_{3}=-0.1 \pm 0.03\right)$ (III) Values of our model. The region of parameters :

$0.0279<4 \epsilon_{1}-2 \epsilon_{2}<0.0670,-0.0471<\frac{4}{3} \epsilon_{1}+\frac{2}{3} \epsilon_{2}<-0.0174,-0.112<\epsilon_{2}+\epsilon_{3}<-0.0902$,

$-0.0925<\epsilon_{3}<-0.0702,4 \epsilon_{1}-2 \epsilon_{2}-3 \epsilon_{2}^{\prime}=-0.108,4 \epsilon_{1}-2 \epsilon_{3}-3 \epsilon_{3}^{\prime}=0.235$,

(We took the center value to $\epsilon^{\prime}$ ).

where we expressed $\rho$-meson propagater in the process $\eta^{\prime} \rightarrow \rho^{0} \gamma \rightarrow \pi^{+} \pi^{-} \gamma$ by using the decay width of the $\rho$-meson $\Gamma_{\rho}$.

\section{$5 \quad$ Hadronic Anomalous Decays}

In this section, we consider hadronic anomalous decays such as $\Gamma(\omega \rightarrow 3 \pi)$. However, the experimental value is presently available only for $\Gamma(\omega \rightarrow 3 \pi)$. As the previous section, we obtained Table 2 for the models of (I) (III).

In Table 2, we took $K^{*} K \pi$-coupling as about $1.05 \times g_{K^{*} K \pi}$, which is given by (2.1), considering $\Gamma\left(K^{* \pm} \rightarrow(K \pi)^{ \pm}\right)=49.8 \pm 0.8 \mathrm{MeV}, \Gamma\left(K^{* 0} \rightarrow(K \pi)^{0}\right)=$ $50.5 \pm 0.6 \mathrm{MeV}$.

As to $\Gamma(\omega \rightarrow 3 \pi)$, we would have to consider the effects of $\left(V P^{3}\right)$-terms from $\Delta \mathcal{L}_{1,2,5}$. But their contributions seem to be very small compared with the contributions from $\Delta \mathcal{L}_{V V P}^{a, b}$, because our prediction of $\Gamma(\omega \rightarrow 3 \pi)$ is already consistent with the experimental value. Therefore, it is sufficient to introduce the isospin/SU(3)-breaking terms only for $\Delta \mathcal{L}_{V V P}^{a, b}$.

In Table 2 it is again suggested that isospin breaking terms are very important. As in the previous section, let us take some expressions cancelling $g$, i.e.,$\Gamma(\omega \rightarrow$ $3 \pi) / \Gamma(\rho \rightarrow 2 \pi)^{3}$. Then we find that predictions of the original $\mathcal{L}_{\text {FKTUY }}$ and 
Bramon et al.[8] are again different from the experiments.

$$
\begin{aligned}
& \frac{\Gamma(\omega \rightarrow 3 \pi)}{\Gamma(\rho \rightarrow 2 \pi)^{3}}= \frac{81 M_{\omega} M_{\rho}^{6}\left(1+\epsilon_{1}+\epsilon_{2}\right)^{2}}{256 \pi^{4} f_{\pi}^{2} P_{\rho \rightarrow \pi \pi}^{9}} \int d E_{+} d E_{-}\left[\boldsymbol{p}_{+}^{2} \boldsymbol{p}_{-}^{2}-\left(\boldsymbol{p}_{+} \cdot \boldsymbol{p}_{-}\right)^{2}\right] \times \\
&\left(\frac{1}{\left(p_{0}+p_{+}\right)^{2}+M_{\rho}^{2}}+\frac{1}{\left(p_{+}+p_{-}\right)^{2}+M_{\rho}^{2}}+\frac{1}{\left(p_{-}+p_{0}\right)^{2}+M_{\rho}^{2}}\right)^{2}(5 . \\
&= 2.38 \times 10^{-6} \mathrm{MeV}^{-2}\left(\text { from (I) and (II) with } \epsilon_{1}=\epsilon_{2}=0\right) \\
& {\left[(2.16 \pm 0.09) \times 10^{-6} \mathrm{MeV}^{-2} \text { from exp. }\right] } \\
& {\left[(2.25 \pm 0.06) \times 10^{-6} \mathrm{MeV}^{-2} \text { from (III) Ours }\right] }
\end{aligned}
$$

Although the only upper bound of $\Gamma(\rho \rightarrow 3 \pi)$ and $\Gamma\left(K^{*} \rightarrow K \pi \pi\right)$ is available now, it will be interesting that their value will be determined by the experiments in future.

\section{Summary}

By introducing isospin/SU(3)-broken $\Delta \mathcal{L}_{V V P}^{a, b}$ with a few parameters, we have shown that each decay width of anomalous process can be consistently reproduced with all the experimental data.

We also made predictions:

\begin{tabular}{rlll}
\hline Decay Mode & & \multicolumn{1}{c}{ Width $(\mathrm{keV})$} & Branching Ratio \\
\hline$\Gamma\left(\rho^{0} \rightarrow \pi^{0} \gamma\right)$ & $=114 \pm 7$ & $(7.54 \pm 0.47) \times 10^{-4}$ \\
$\Gamma\left(\phi \rightarrow \eta^{\prime} \gamma\right)$ & $=0.552 \pm 0.055$ & $(1.25 \pm 0.13) \times 10^{-4}$ \\
$\Gamma\left(\rho^{0} \rightarrow \pi^{0} \pi^{+} \pi^{-}\right)$ & $=6.70 \pm 3.25$ & $(4.43 \pm 2.15) \times 10^{-5}$ \\
$\Gamma\left(\rho^{ \pm} \rightarrow \pi^{ \pm} \pi^{0} \pi^{0}\right)$ & $=4.76 \pm 1.70$ & $(3.15 \pm 1.12) \times 10^{-5}$ \\
$\Gamma\left(\rho^{ \pm} \rightarrow \pi^{ \pm} \pi^{+} \pi^{-}\right)$ & $=0.125 \pm 0.121$ & $(8.28 \pm 8.00) \times 10^{-7}$ \\
$\Gamma\left(K^{*-} \rightarrow \bar{K}^{0} \pi^{0} \pi^{-}\right)$ & $=12.3 \pm 1.1$ & $(2.47 \pm 0.22) \times 10^{-4}$ \\
$\Gamma\left(K^{*-} \rightarrow K^{-} \pi^{+} \pi^{-}\right)$ & $=6.26 \pm 0.55$ & $(1.26 \pm 0.11) \times 10^{-4}$ \\
$\Gamma\left(K^{*-} \rightarrow K^{-} \pi^{0} \pi^{0}\right)$ & $=0.685 \pm 0.081$ & $(1.38 \pm 0.16) \times 10^{-5}$ \\
$\Gamma\left(\bar{K}^{* 0} \rightarrow K^{-} \pi^{0} \pi^{+}\right)$ & $=16.8 \pm 1.6$ & $(3.33 \pm 0.32) \times 10^{-4}$ \\
$\Gamma\left(\bar{K}^{* 0} \rightarrow \bar{K}^{0} \pi^{-} \pi^{+}\right)$ & $=6.26 \pm 0.60$ & $(1.24 \pm 0.12) \times 10^{-4}$ \\
$\Gamma\left(\bar{K}^{* 0} \rightarrow \bar{K}^{0} \pi^{0} \pi^{0}\right)$ & $=0.522 \pm 0.05$ & $(1.03 \pm 0.10) \times 10^{-5}$ \\
\hline
\end{tabular}

Table 3: The List of Our Predictions

We expect that the decay data for pseudoscalar mesons and vector mesons, such as $\phi \rightarrow \eta^{\prime} \gamma, \rho^{0} \rightarrow \pi^{0} \gamma$ etc., will be obtained with good accuracy in the DA $\Phi$ NE $\phi$-factory. 


\section{Acknowledgements}

The author is very grateful to K. Yamawaki for suggesting this subject, helpful discussions and also for careful reading the manuscript. Thanks are also due to M. Sugiura, A. Shibata and others in the laboratory at Nagoya University for discussions and encouragement.

\section{[Note added]}

After completion of this work, the author was informed by M. Harada that a similar analysis has been done by M. Harada and J. Schechter who, however, assumed the breaking term is proportional to the quark mass matrix and thereby arrived at inconsistency with the data.

\section{References}

[1] A. Bramon, A. Grau and G. Pancheri, in "Second DA $\Phi$ E Physics Handbook", eds. L. Maiani, L. Pancheri and N. Paver (INFN, Franzini, 1995), p. 477;

S. I. Eidelman, ibid, p. 523;

J. Lee-Franzini, ibid, p. 761.

[2] Particle Data Group, Phys. Rev. D50 (1994) 1173.

[3] J. Wess and B. B. Zumino, Phys. Lett. 37B (1971) 95.

E. Witten, Nucl. Phys. B223 (1983) 422.

[4] M. Bando, T. Kugo, S. Uehara, K. Yamawaki, and T. Yanagida, Phys. Rev. Lett. 54 (1985) 1215.

[5] M. Bando, T. Kugo and K. Yamawaki, Nucl. Phys. B259 (1985) 493;

[6] T. Fujiwara, T. Kugo, H. Terao, S. Uehara and K. Yamawaki, Prog. Theor. Phys. 73 (1985) 926.

[7] M. Bando, T. Kugo and K. Yamawaki, Phys. Rep. 164 (1988) 217.

[8] A. Bramon, A. Grau and G. Pencheri, Phys. Lett. B344 (1995) 240.

[9] D. Alde et al., Z. Phys. C61 (1994) 35.

[10] M. Harada and J. Shecheter, hep-ph/9506473. 
[11] J. Gasser and H. Leutwyler, Ann. Phys. 158 (1984) 142.

J. Gasser and H. Leutwyler, Nucl. Phys. B250 (1985) 465.

[12] J. Gilman and R. Kauffman, Phys. Rev. D36 (1987) 2761. 